\title{
Reversible Data Hiding in Digital Image
}

\author{
Karpagaselvi Subramanian, S.Vairachilai
}

\begin{abstract}
The growth of multimedia technologies and the attractiveness of internet are dramatically increasing. All kinds of multimedia information like audio, video and images can be obtained freely and copied even edited then transmitted as one wishes. At the same time, this makes the integrity, reliability, and security of data transmitted under threat. The information security has always been a major concern. As one of the ways to solve the security problem is data hiding technology that embeds the secret data imperceptibly into the cover media by slightly modifying some of the cover elements. This paper discusses the basic principles of reversible data hiding (RDH) as a method of covert communication in order to understand how information is embedded in the graphical representation and covers the in-depth discussion on image compression.
\end{abstract}

Key words: Reversible data hiding, JPEG, Compression, discrete cosine transform, Quantization.

\section{INTRODUCTION}

Traditional data hiding techniques usually introduce irreversible distortion in the cover media which cannot be recovered after secret data extraction. However, the distortion may be not allowed in the medical, military and hiding has been proposed as a new solution where both secret data and the original cover media can be extracted and recovered without any distortion.Moreover, data hiding techniques can be applied to other aspects such as embedding patient's information into medical images and embedding geographic information into remote sensing satellite images [1].

A flurry of research works has been contributed by researchers about lossless data hiding scheme for Joint Photographic Experts Group (JPEG) images within in the last few years. Some of the data hiding techniques on JPEG images uses standard quantization table to embed data into an image while others embed data by modifying the quantization table. The JPEG images processing algorithms vary according to the purpose of embedding. Some uses lossless algorithms while others use reversible watermarking embedding techniques. In the lossy algorithms, the embedded data will be recovered where as in the lossless techniques both the message and the data will be recovered [2]. In reversible data hiding in JPEG, the discrete cosine transform (DCT) coefficients is based on four general data hiding approaches [2].

The first one is lossless compression based which was proposed by Fridrich [3] for secret data embedding. In this approach, a space is preserved by compressing the redundant component of the image. Since message capacity is too small, this method takes less attention of researchers.

Revised Manuscript Received on 14 September, 2019.

Karpagaselvi Subramanian, Mearg Enqubahri School of Electrical and Computer Engineering Ethiopian Institute of Technology-Mekelle Mekelle University Ethiopia.

S.Vairachilai, Department of Computer Science and Engineering Faculty of Science and Technology, The ICFAI Foundation for Higher Education, Hyderabad, India. legal fields. To overcome this problem, reversible data

The second approach is quantization table modification approach which was proposed by Fridrich [4] to authenticate digital image. Later, Wang et al. [5] proposed a method which is based on modifying the quantization table and quantized DCT coefficients. In this approach, some elements of the quantization table are divided by an integer while the corresponding quantized DCT coefficients are multiplied by the same integer and added by an adjustment value to make space for embedding the data. This result shows an improvement on the Peak Signal to Noise Ratio (PSNR) value over previous works. This approach is basically works by preprocessing the quantized DCT coefficients to create space for secret data embedding. This is done by making the selected DCT coefficients a multiple of an integer $\mathrm{k}$. If D is quantized DCT coefficients then D' (preprocessed DCT coefficients) is multiple of D and k, where $\mathrm{k} \in\{2,3 \ldots\}$. The corresponding quantization table entry again divided by $\mathrm{k}$ to compensate multiplication side effect on image distortion. The remainders of the quantization table division padded to the main message as side information so that decoder will use it to construct the original values. The binary secret message is changed into $k$ 's based number format is another part of the method to have high capacity embed ability efficiency. Finally, according to the embedding algorithm encoder can embed secret data in the modified DCT values. Using the predefined integer, decoder can easy apply modulo operation on modified DCT coefficients to get the embedded secret message, divide DCT coefficients and multiply quantization table entries to get the original DCT and quantization table values respectively.

The third method is based on modification of Huffman table which was proposed by Mobasseri et al. [6] In this method, data embedding is performed by mapping variable length code (VLC) to an unused VLC. The Qian and Zhang's scheme [2] is another research work related to data embedding on JPEG images. The work can be seen from the point of JPEG bit stream structure and the scheme perspectives. From the JPEG bit stream structure perspective, an input gray-scale image is compressed into the JPEG format by the JPEG encoder and saved in the form of bit stream. It starts with an Start of Image (SOI) marker and ends with an End of Image (EOI) marker. The Huffman data is used to generate the Huffman table. The scheme is based on Mobasseri et al.'s method which works by flipping one or more bits of the non-zero AC Coefficients. This work also improved of mobasseri et al.'s method [6], but still the payload is too small.

The fourth method is based on modifying the quantized DCT coefficients. This coefficients modification approach is proposed by Chang et al. [7] and Xuan et al. [8]. Sakai et al. [9] improved Xuan et al. [8] method by block selection approach and get better visual quality of the image. Li et al.

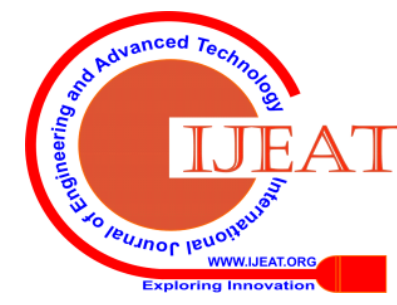




\section{Reversible Data Hiding in Digital Image}

[10] proposed reversible data hiding scheme on JPEG images based on smaller DCT value selection and slightly modification of quantization table. Based on histogram shift (HS) strategy, Lin et al. [11] discussed high capacity reversible data hiding for JPEG image. They used different block sizes to get high embedding capacity. Celik et al. [12] proposed lossless data hiding in JPEG DCT coefficients least significant bit (LSB). In [12], the DCT value which has high distortion is padded as side information to the main secret message. Wang et al. [1], Xuan et al. [8] and Sakai et al. [9] are done the best research works on reversible data hiding for JPEG images based on quantization table and DCT values modification scenario. Although the experimental result of [1] show better PSNR, the file size increate a lot because of multiplication operation. If $\mathrm{D}=15$ and $\mathrm{k}=3$, then $\mathrm{D}$ ' becomes 45 . The memory space used to store $\mathrm{D}^{\prime}$ ' is 2 bit more compared to D. Shifts the quantized DCT coefficient histogram with an optimum searching strategy. This optimum strategy used to achieve good performance. In order to make data embedding unperceivable and the visual quality of marked image higher when embedding a certain amount of data, only lower and mid-frequency DCT coefficients are chosen to embed data in the embedding process. Sakai et al. [9] improved Xuan et al.'s scheme in 2008. This method yielded better image quality based on new block selection strategy.

\section{DATA HIDING}

Data hiding is a group of techniques used to keep a secure data in a host media with small deterioration in host that can be used to extract the same data in later on process. Steganography is one such pro-security innovation in which secret data is embedded in a cover [13]. This method fall into reversible data hiding. In reversible data-hiding, the information bits are inserted by modifying the host signal that will enable the lossless restoration of the original host signal after extracting the embedded information. However, the possibility of recovering the exact original image is a desirable property in many fields such as legal, medical, and military imaging.

The documents like bank checks are scanned \& protected with an authentication scheme based on a reversible data hiding and sent through the Internet. In most cases, the watermarked documents will be sufficient to distinguish unambiguously the contents of the documents. However, if any uncertainty arises, the possibility of recovering the original unmarked document is very interesting [14]. The block diagram of a basic reversible data hiding system shown in Figure 1. The existing reversible data hiding schemes are mostly fragile in nature. The two important properties of reversible data hiding are imperceptibility and embedding capacity. The imperceptibility is the measure of similarity between stego and the cover image. The embedding capacity is the measure of the maximum number of information bits that can be embedded in the cover image. The performance of a reversible data hiding technique is evaluated on the basis of imperceptibility and embedding capacity.

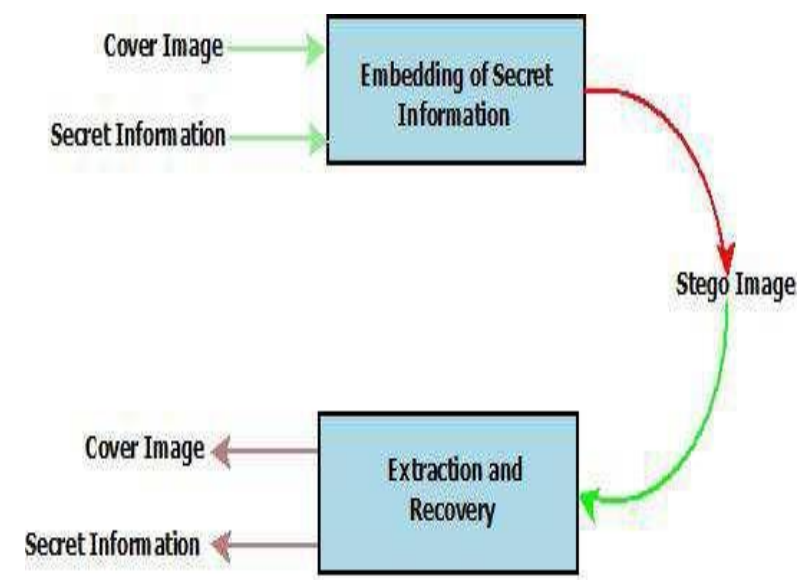

Figure 1: Block diagram of basic reversible data hiding system [[13]

\section{REVERSIBLE DATA HIDING TeCHNIQUeS}

Data hiding techniques are characterized by the embedding and extraction techniques. The reversible data hiding techniques classified into compression based, histogram modification based, quantization based, and expansion based techniques. The RDH techniques are getting more popular because of its increasing sensitive applications areas such as military communication, healthcare, and law- enforcement [13].

In the medical imagery field, if a medical image is modified using conventional watermarking, the small change may affect the interpretation significantly and a physician may make a wrong diagnosis. In military application, the changes due to embedding of secret information can substantially alter the cover image and therefore, the decision taken may cost considerably. Consequently, there is a strong need to restore the cover work to its original form. The reversible data hiding known as lossless data hiding that allows full extraction of the embedded information along with the complete restoration of the cover work. The reversible data hiding can be considered as a special case of steganography.

\section{A. Compression based $\mathrm{RDH}$}

In order to recover the original image, the information is stored along with the secret data. In case of $\mathrm{RDH}$, additional data needs to be embedded. In addition, it needs more space compared to conventional data hiding for data embedding. A simple approach will compress a part of cover image for embedding data. Several reversible data hiding schemes are reported using this approach [15]. In this approach, image is first transformed into frequency domains by taking integer wavelet transform and then dividing into blocks. The compression operation is performed on each coefficient of a block whose value is greater than a certain threshold. Each block has its own threshold value.

\section{B. Histogram Modification Based RDH}

In this type of $\mathrm{RDH}$, histogram of the image in pixel/transform domain is calculated. Then, based on the histogram plot the schemes try to embed data by changing the plot of original histogram.

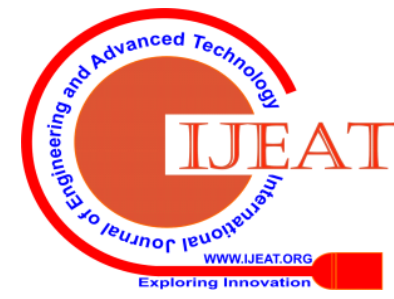


This is based on the concept of histogram shifting. Ni et al. [16] proposed a reversible data hiding method which shifts slightly the part of the histogram between the maximum point (peak point) and the minimum point to the right side by one pixel value to create an empty bin besides the maximum point for hiding an input message. This method yields hiding capacities and provides higher qualities in stego-images. The knowledge of the maximum point and the minimum point of the histogram is necessary for retrieving the hidden data and restoring the stego-image losslessly to the original state. In this method, $P$ is the value of peak point and $\mathrm{Z}$ is the value of zero point. The range of the histogram $\mathrm{P}+1 \& \mathrm{Z}-1$ is shifted to the right-hand side by 1. Once a pixel with value $P$ is encountered, if the message bit is set as 1 , the pixel value is increased by 1 otherwise no modification is needed.

The data extraction is the reverse of the data hiding process. In this approach, first, image is divided into several blocks of neighboring pixels and then each block is split into two zones. Finally, the corresponding histograms are calculated. A bin is shifted in accordance with the value of corresponding secret bit. If the bit is 1 , shift the lowest bin to the highest one and downgrade other bins. If the bit is 0 , upgrade each bin and shift the highest bin to the lowest bin. $\mathrm{Ni}$ et al. [16] utilizes zero or minimum point of histogram. If the peak is lower than the zero or minimum point in the histogram, it increases pixel values by one from higher than the peak to lower than the zero or minimum point in the histogram. While embedding, the whole image is searched. Once a peak-pixel value is encountered, if the bit to be embedded is '1' the pixel is added by 1 , else it is kept intact. Alternatively, if the peak is higher than the zero or minimum point in the histogram, the algorithm decreases pixel values by one from lower than the peak to higher than the zero or minimum point in the histogram and to embed bit ' 1 ' the encountered peak-pixel value is subtracted by 1 .

\section{Difference Expansion Based RDH}

Tian [17] proposed a high quality reversible watermarking method with high capacity based on difference expansion. The pixel differences are used to embed the data because of high redundancies among the neighboring pixel values in natural images. The neighboring pixel values are calculated during the embedding process and the changeable bits differences are determined. Some differences are chosen to be expandable by 1-bit, the changeable bits increases, concatenated bit-stream of compressed original changeable bits, the location of expanded difference numbers (location map) and the hash of original image (payload) is embedded into the changeable bits of difference numbers in a pseudo random order. This used the inverse transform to have the watermarked pixels from resultant differences. This method is good in video and audio but it suffers significant degradation of visual quality due to bit-replacements of gray scale pixels. This method depends on the smoothness of natural image. It cannot be applied to textured image [18].

\section{Quantization based RDH}

Quantization based data hiding techniques are robust. However, the reversible quantization based data hiding approaches are mostly fragile in nature. In this method, embedding a message into a host signal. This refers to quantization of the host signal by a quantizer picked from an ensemble of quantizer where each quantizer is associated with a message letter/index. One of the relevant approaches is Sequential Quantization Strategy (SQS) for data embedding. The SQS makes the modulation of a pixel value dependent on the previous pixels. A reversible data hiding method is used with SQS to make it more suitable for the authentication purposes. This algorithm ensures the recovery of cover image [14] for medical imaging systems.

\section{E. DCT Based Data Hiding}

For the past few years, a standardization effort known by the acronym JPEG $[19,6,20]$ has been working toward establishing the first international digital image compression standard for continuous-tone (multilevel) still images both grayscale and color. The discrete cosine transform is a general orthogonal transform for digital image processing and signal processing. The advantages of this method are small bit error rate, good information integration ability, good synthetic effect of calculation complexity and high compression ratio [21]. This scheme also requires a preprocessing phase because the secret data are hidden in the DCT coefficients of a cover image. In preprocessing phase, first the JPEG image partitioning into non-overlapping blocks of $8 \times 8$ blocks. Second, the DCT of each block are computed and the resultant DCT coefficient matrix is quantized using a standard quantization table. Finally, the inverse DCT of quantized coefficient matrix are evaluated and the final JPEG image is obtained after rounding the values [4]. The key steps of JPEG compression process [19, $22,20]$ is shown in Figure 2. The color image can be approximately regarded as multiple grayscale images. The JPEG encoder (DCT based encoder) consists of three parts: (i) DCT (ii) Quantizer (iii) Entropy coder. First, the original image is divided to $8 \times 8$ non-overlapping blocks. Second, each block will be transformed from spatial domain to frequency domain using two-dimensional DCT function. In the quantization phase, the obtained DCT coefficients are divided by the predetermined quantization table. The quantized DCT coefficients are arranged in a zigzag order. After scanning the DCT coefficients pre-compressed using the differential pulse code modulation (DPCM) on DC coefficients and run length encoding (RLE) on AC coefficients. Finally, the symbol string is Huffman coded to obtain the final compressed bit-stream. After pre-pending the header, the final JPEG file is obtained.

$$
\text { Quantized Walue }\left(u_{n} W\right)=\frac{\operatorname{DCT}\left(u_{n}, W\right)}{\text { Quantum }\left(w_{n} w\right)}
$$




\section{Reversible Data Hiding in Digital Image}

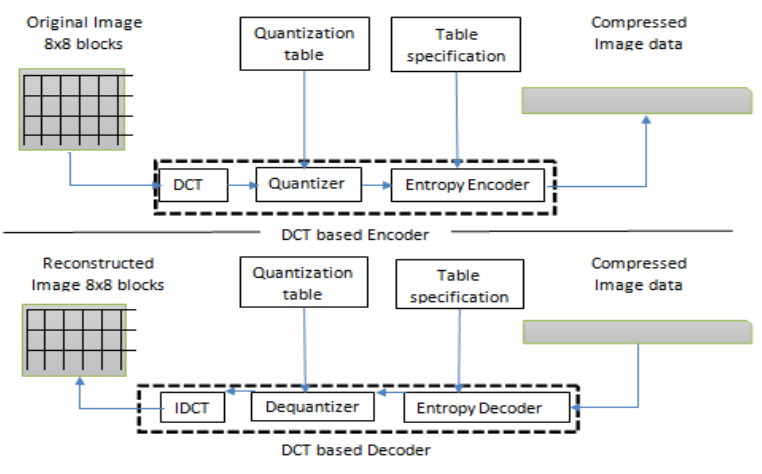

Figure 2: The block diagram of JPEG compression process

\section{JPEG COMPRESSION}

JPEG [23] format is one of the most popular formats and widely used on various platforms [1]. For instance, an uncompressed $640 * 480$ pixel 24-bit color image would require 900 kilobyte $(\mathrm{KB})$, whereas a JPEG version of the same image can be compressed to $150 \mathrm{~KB}[24]$. In data hiding there are two common methods of embedding: (i) Spatial domain method [23] in which messages are inserted into the LSBs of image pixels (ii) Variable bit LSB. In LSB substitution, images directly embedded into the pixels of the cover image by using 1bit-LSB, 2bit-LSB, Variable bit LSB. In the frequency-domain $[16,23]$ the secret image is first transformed to frequency-domain, and then the messages are embedded in the transformed coefficients. Transform embedding methods are more robust than the spatial embedding methods which are susceptible to imageprocessing type of attacks. The discrete cosine transform [16] is a widely used tool for frequency transformation. If we apply JPEG images to data hiding, the stego-image will not easily draw attention of suspect. The DCT methods gives minimum distortion between the original cover and stego images while Walsh gives the maximum distortion. DCT is the most efficient transform for embedding [23].

\section{A. Co-efficient Quantization}

The DCT output matrix takes more space to store than the original matrix of pixels. The input to the DCT functions consists of eight bit pixels values. The values range from a low -1024 to a high 1023, occupying eleven bits. To discard an appropriate amount of information, the encoder divides each DCT output value by a quantization value and rounds the result to an integer. A quantization value of sixteen produces a quantized DCT co-efficient with seven bit precision. If the quantization value is large then the more data is lost. The actual DCT value is represented less accurately. Quantization is simply the process of reducing the number of bits needed to store an output value by reducing the number of bits to represent integer results. Each of the 64 positions in the $8 \times 8$ matrix has its own quantization value with the higher order terms being quantized more heavily than the low-order terms. Thus, the higher-order terms have larger quantization values. In the quantization table/ matrix, for every element position in the DCT matrix has a corresponding quantum value. The quantum value indicates what the step size is going to be for that element in the compressed rendition of the picture with values ranging from 1 to 255 . The elements to the picture will be encoded with a small step size, the size one offering the most precision. The values can become higher as they move away from the origin. The formula for quantized is as follows:

JPEG image compression is based on the DCT, which is one of the basic building blocks for JPEG compression [19, 22]. The important aspect of DCT for JPEG compression is the ability to quantize the DCT coefficients using visually weighted quantization values. JPEG standard does not specify the DCT quantization table that everybody (researchers) can use their own quantization table by scaling the table elements using different quality factor [19, 22]. The different quantization table used the different image quality and compression ratio. The most known quantization table used by many researchers is shown in Table 1 .

A scaling factor (SF) is computed with Equation (2) with the input Quality factor (QF) which is a positive integer number up to $100, \mathrm{QF}=\{1,2 \ldots 100\}$ is used to scale the quantization table values [1]. The scaled quantization table is obtained using Equation (1). The scaled quantization matrix[1] is then rounded and clipped to have positive integer values ranging from 1 to 255 using Equation (3).

The $8 \times 8$ block of DCT coefficients is compressed by quantization. The quantization is achieved by dividing each element in the DCT coefficient block by the corresponding value in the quantization matrix, and the result is rounded to the nearest integer. A useful feature in this process is the image compression. The quality is obtainable through selection of specific quantization table. The DCT block consists of 64 DCT coefficients. The top-left coefficients $(0,0)$ the original image block, which is called Direct current (DC) coefficient. As we move away from the $\mathrm{F}(0,0)$ in all directions the DCT coefficients are Alternative current (AC) coefficients.

$$
\begin{aligned}
& S E= \begin{cases}\underline{5000}, & \text { if } Q F<50 \\
Q F & \\
200-(2 * Q F), & \text { if } Q f \geq 50\end{cases} \\
& Q_{\text {scatiod }}(i, j)=\operatorname{MAX}\left(\operatorname{round}\left(Q_{\text {mmatal }}(i, j) * \frac{S F}{)}\right)||\right)
\end{aligned}
$$

selection of specific quantization table. The DCT block consists of 64 DCT coefficients. The top-left coefficients $(0,0)$ the original image block, which is called Direct current (DC) coefficient. As we move away from the $F(0,0)$ in all directions the DCT coefficients are Alternative current (AC) coefficients.

\section{A. Zigzag sequence}

After the quantization, the next step in the JPEG algorithm is to entropy encoding of the quantized DCT coefficients. The DC coefficients are a measure of the average value of the 64 image samples. Because there is usually a strong correlation between the DC coefficients

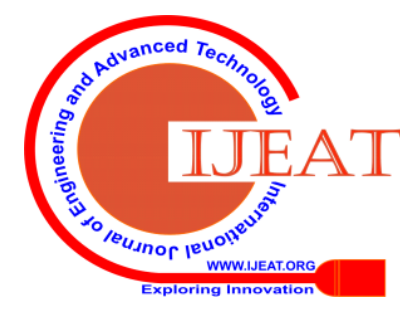


TABLE 1. Standard Quantization Table ( Qtan $)$

\begin{tabular}{|c|c|c|c|c|c|c|c|}
\hline 10 & \begin{tabular}{c}
7 \\
\hline 7
\end{tabular} & 6 & 10 & 14 & 4 & 31 & 37 \\
\hline 8 & 8 & 10 & 14 & 24 & 34 & 41 & 34 \\
\hline 8 & 10 & 13 & 17 & 31 & 52 & 48 & 37 \\
\hline 11 & 13 & 22 & 34 & 41 & 65 & 62 & 46 \\
\hline 14 & 21 & 33 & 38 & 49 & 62 & 68 & 55 \\
\hline 29 & 38 & 47 & 52 & 62 & 73 & 72 & 61 \\
\hline 43 & 55 & 57 & 59 & 67 & 60 & 62 & 59 \\
\hline
\end{tabular}

TABLE 2: $\quad$ Scaled Quantization Table $(\mathrm{QF}=70)$

\begin{tabular}{|l|l|l|l|l|l|l|l|}
\hline 16 & 11 & 10 & 16 & 24 & 40 & 51 & 61 \\
\hline 12 & 12 & 14 & 19 & 26 & 58 & 60 & 55 \\
\hline 14 & 13 & 16 & 24 & 40 & 57 & 69 & 56 \\
\hline 14 & 17 & 22 & 29 & 51 & 87 & 80 & 62 \\
\hline 18 & 22 & 37 & 56 & 68 & 109 & 103 & 77 \\
\hline 24 & 35 & 55 & 64 & 81 & 104 & 113 & 92 \\
\hline 49 & 64 & 78 & 87 & 103 & 121 & 120 & 101 \\
\hline 72 & 92 & 95 & 98 & 112 & 100 & 103 & 99 \\
\hline
\end{tabular}

The $8 \times 8$ block of DCT coefficients is compressed by quantization. The quantization is achieved by dividing each element in the DCT coefficient block by the corresponding value in the quantization matrix, and the result is rounded to the nearest integer. A useful feature in this process is the image compression. The quality is obtainable through from the DC term of the previous block in the encoding order. The DC coefficients frequently contain a significant fraction of the total image energy. Finally, all quantized AC coefficients are ordered into a zigzag sequence that shown in Table 2. This ordering of coefficient helps to facilitate entropy encoding by placing the low frequency coefficients (which are more highly to be non-zero) before high frequency components.

\section{CONCLUSION}

Reversible data hiding techniques are used to hide secrete information inside cover image, as well as to recover the cover image after extraction of secrete message. RHD can be applied on encrypted image so that others do not know the original content. In this paper, we have reviewed several methods to understand how information is embedded in the graphical representation. A survey on various reversible data hiding techniques and image compression are discussed. In future, it is essential to develop an efficient method that provides data embedding and recovery without any distortion and provides better security

\section{REFERENCES}

1. Wang, Kan, Zhe-Ming Lu, and Yong-Jian $\mathrm{Hu}$, "A high capacity lossless data hiding scheme for JPEG images," Journal of Systems and Software, Vol. 86, No. 7, pp. 1965-1975, 2013.
2. Qian, Zhenxing, and Xinpeng Zhang, "Lossless data hiding in JPEG bitstream," Journal of Systems and Software, Vol. 85, No. 2, pp. 309$313,2012$.

3. Fridrich, Jessica, Miroslav Goljan, and Rui Du, "Lossless data embedding for all image formats," International Society for Optics and Photonics, Vol. 4675, pp. 572-583, 2002.

4. ridrich, Jiri, Miroslav Goljan, and Rui Du, "Invertible authentication watermark for JPEG images," Proc. Information Technology: Coding and Computing, IEEE Xplore, pp.223-227, DOI:10.1109/ITCC.2001.918795, 2001.

5. Wang, Kan, Zhe-Ming Lu, and Yong-Jian Hu, "A high capacity lossless data hiding scheme for JPEG images," Journal of Systems and Software, Vol. 86, No. 7, pp. 1965-1975, 2013.

6. Mobasseri, Bijan G, Robert J.Berger, Michael P.Marcinak, and Yatish J.NaikRaikar. "Data embedding in JPEG bitstream by code mapping," IEEE Transactions on Image Processing, Vol. 19, No. 4, pp. 958-966, 2010.

7. Chin-Chen Chang, Chia-Chen Lin, Chun-Sen Tseng, and Wei-Liang Tai,"Reversible hiding in DCT-based compressed images," Information Sciences, Vol. 177, No. 13, pp. 2768-2786, 2007.

8. Xuan, Guorong, Yun Q. Shi, Zhicheng Ni, Peiqi Chai, Xia Cui, and Xuefeng Tong, "Reversible data hiding for JPEG images based on histogram pairs," Image Analysis and Recognition, Lecture Note in computer science, Vol. 4633, pp. 715-727, 2007.

9. Sakai, Hirofumi, Minoru Kuribayashi, and MasakatuMorii, "Adaptive reversible data hiding for JPEG images," Information Theory and Its Applications, IEEE International Symposium, pp. 1-6, DOI: 10.1109/ISITA.2008.4895529, 2008

10. Li, Qiming, Yongdong Wu, and Feng Bao, "A reversible data hiding scheme for JPEG images," In Advances in Multimedia Information Processing-PCM, vol. 6297, Lecture Note in computer science, pp. 653-664. 2010.

11. Lin, Yih-Kai, "High capacity reversible data hiding scheme based upon discrete cosine transformation." Journal of Systems and Software, Vol. 85, No. 10, pp. 2395-2404, 2012.

12. Celik M.U, Gaurav Sharma, Murat Tekalp A, and Eli Saber, "Lossless generalized-LSB data embedding," IEEE Transaction on Image Processing, Vol. 14, No. 2, pp. 253-266, 2005.

13. Pooja Prabhakar Petkar and P. S. Nalwade, "A Survey on Reversible Data Hiding Techniques," International of Computer Technology \& Applications, Vol 5,pp. 897-903, 2014.

14. Vaishnavi, V. and Kuechler, W, "Design Science Research in Information Systems" Auerbach Publications Boston, MA, USA, 2007.

15. Ankita Meenpal and Shital S Mali, "Survey of Recent Reversible Data Hiding Schemes," International Journal of Advance Foundation and Research in Computer, Vol 1, pp.2348 - 4853, 2014.

16. Ni, Zhicheng, Shi Qing, Nirwan Ansari and Wei Fang Su, "Reversible data hiding," IEEE Transaction on Circuits and Systems for Video Technology, Vol. 16, No. 3, pp. 354-362, 2006

17. Tian, Jun, "Reversible data embedding using a difference expansion," IEEE Transaction on Circuits System for Video Technology, Vol. 13, No. 8, pp. 890-896, 2003.

18. Mohammad Awrangjeb, "An Overview of Reversible Data Hiding,"Conference: International Conference on Computer and Information Technology, pp 75-79, 2003.

19. Pennebaker, William B., and Joan L. Mitchell. JPEG: Still image data compression standard. Springer Science \& Business Media, 1993.

20. Suhad Shakir Zahereel I. and Abdul Khalib," Survey On Recent Digital Image Steganography Techniques" Journal of Theoretical and Applied Information Technology, Vol. 66 No.3,2014.

21. Blossom Kaur, Amandeep Kaur, and Jasdeep Singh, "Steganographic approach for hiding image in dct domain," International journal of advances in engineering \& technology, pp. 2231-1963, 2011.

22. Wallace, Gregory K, "The JPEG still picture compression standard," Communications of the ACM, Vol. 34, No. 4, pp. 30-44, 1991.

23. Xiaochao $\mathrm{Qu}$, and HyoungJoong Kim, "Pixel-based pixel value ordering predictor for high-fidelity reversible data hiding," Signal Processing, Vol. 111, pp. 249-260, 2015.

24. Jesse D. Kornblum, "Using JPEG quantization tables to identify imagery processed by software," Digital investigation Vol.5, pp. 21$25,2008$.

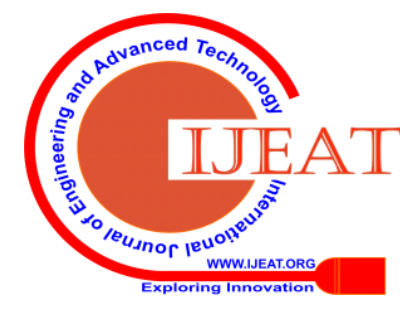

